\title{
Revision of measures to combat intractable diseases in Japan: Three pillars will play an even greater role in the future
}

\author{
Peipei Song, Norihiro Kokudo* \\ Department of Surgery, Graduate School of Medicine, The University of Tokyo, Tokyo, Japan.
}

Keywords: Rare diseases, orphan drugs

\begin{abstract}
Summary: Over the past 40 years, measures to combat intractable diseases in Japan have progressed substantially since the implementation of the "Outline of Measures to Combat Intractable Diseases" in 1972. However, many challenges remain. In order to further promote measures to combat intractable diseases, a "Revision of Measures to Combat Intractable Diseases" was approved by the Japanese Ministry of Health, Labor, and Welfare (MHLW) on January 25, 2013. The revision rests on the three pillars of development of effective strategies to treat intractable diseases and improved care for those affected, creation of fair and consistent mechanisms to reimburse medical expenses, and implementing measures to enhance public understanding and encourage the social participation of those affected. These pillars will play an even greater role in future measures to combat intractable diseases.
\end{abstract}

On January 25, 2013, a "Revision of Measures to Combat Intractable Diseases" was approved by the Japanese Ministry of Health, Labor, and Welfare (MHLW) (1) to further promote future measures to combat intractable diseases.

Intractable diseases (Nanbyo), usually referred to as "rare and intractable diseases" in Japan, are currently defined as"a disease of unknown etiology with no effective treatment that presents a major financial and psychological burden and that is rare (fewer than 50,000 total patients)" (2). The "Outline of Measures to Combat Intractable Diseases" was implemented in 1972. Since then, measures to combat intractable diseases in Japan have progressed substantially, including the "Specified Disease Treatment Research Program" established in 1972 with the support of the

\section{*Address correspondence to:}

Dr. Norihiro Kokudo, Department of Surgery, Graduate School of Medicine, The University of Tokyo, 7-3-1 Hongo, Bunkyo-ku, Tokyo 113-8655, Japan.

E-mail: KOKUDO-2SU@h.u-tokyo.ac.jp
MHLW to promote research on 130 intractable diseases, the revised "Orphan Drug Regulation" enacted in 1993 to encourage discovery and development of orphan drugs with specific incentives, the "Intractable Disease Information Center" established in 1997 to provide vast information to patients with intractable diseases, and designation of "Bases for Early and Exploratory Clinical Trials in Specific Research Areas" starting in 2011 to promote the development of innovative drugs and medical devices originating from Japan (2-4). However, many challenges remain, such as the varied needs of patients with intractable diseases and their families, gaps in knowledge of tools and strategies to treat intractable diseases, and reimbursement of the considerable financial burden caused by intractable diseases. Given this situation, a "Revision of Measures to Combat Intractable Diseases" was approved in January 2013 in Japan in order to promote measures to combat intractable diseases in light of changing social and financial resources.

The revision clearly described the current state of intractable diseases in Japan and it identified research programs and future strategies to treat intractable diseases. Revision of the measures to combat intractable diseases rests on three pillars. First is the development of effective strategies to treat intractable diseases and improved care for those affected. It includes four important aspects: governmentfunded special biomedical research programs to enhance basic and applied research on intractable diseases, establishment of a national intractable diseases registry and effective use of databases at the national and international levels, formulation of guidelines and establishment of a support network for specific intractable diseases, and designation of certain hospitals as bases for treatment of intractable diseases. The second pillar is creation of fair and consistent mechanisms to reimburse medical expenses for intractable diseases. Aspects of this pillar include: collection of patient data and establishment of criteria 
defining a specific intractable disease, diagnosis and certification of individuals with intractable diseases, and determination of appropriate levels of benefits. The third pillar is implementing measures to enhance public understanding and encourage the social participation of those affected by intractable diseases. Aspects of this pillar include: educational campaigns on intractable diseases as well as the provision of social welfare services, employment assistance, and counseling to patients' and their families.

Intractable diseases are a pressing public health issue and a challenge to medical care worldwide. The "Revision of Measures to Combat Intractable Diseases" represents a positive perspective on the development of measures to combat intractable diseases in Japan, and this step may serve as a reference for other countries coping with intractable diseases elsewhere around the world.

\section{References}

1. The Japanese Ministry of Health, Labor, and Welfare. Revision of Measures to Combat Intractable Diseases. http://www.mhlw.go.jp/stf/houdou/2r9852000002udoe. html (accessed February 1, 2013).

2. Song PP, Gao JJ, Inagaki Y, Kokudo N, Tang W. Rare diseases, orphan drugs, and their regulation in Asia: Current status and future perspectives. Intractable Rare Dis Res. 2012; 1:3-9.

3. Song P, Gao J, Inagaki Y, Kokudo N, Tang W. Intractable and rare diseases research in Asia. Biosci Trends. 2012; 6:48-51.

4. Song PP, Gao JJ, Kokudo N, Tang W. New opportunity for orphan drug development in Japan: Early exploratory clinical trial bases promote drug translation from basic studies to clinical application. Intractable Rare Dis Res. 2012; 1:95-97.

(Received February 12, 2013) 